Tumour glycolysis: the many faces of HIF

Kelly, C.J. and Smallbone, K. and Brady, J.M. 2008

Manchester Institute for Mathematical Sciences

School of Mathematics

The University of Manchester

\footnotetext{
Reports available from: http://eprints.maths.manchester.ac.uk/

And by contacting: The MIMS Secretary

School of Mathematics

The University of Manchester

Manchester, M13 9PL, UK
} 


\title{
Tumour glycolysis: The many faces of HIF
}

\author{
Catherine Kelly $^{\mathrm{a}, *}$, Kieran Smallbone ${ }^{\mathrm{b}}$, Michael Brady ${ }^{\mathrm{a}}$ \\ a Information Engineering, University of Oxford, Oxford OX1 3PJ, UK \\ ${ }^{\mathrm{b}}$ Manchester Centre for Integrative Systems Biology, University of Manchester, M1 7DN, UK
}

\section{A R T I C L E I N F O}

\section{Article history:}

Received 15 May 2008

Accepted 17 May 2008

Available online 29 May 2008

Keywords:

Molecular imaging

\begin{abstract}
A B S T R A C T
We present a model for tumour metabolism that incorporates both microenvironmental (extracellular) and oncogenic (intracellular) influences. We explore the effects of the interaction between the hypoxic microenvironment and intracellular signalling on the glycolytic response of tumour tissue, finding that the glycolytic state is dependent on a delicately balanced interplay between the cellular hypoxic response, mediated by hypoxia-inducible factor- $1 \alpha$ (HIF- $1 \alpha$ ), and growth-factor signalling cascades, which are frequently mutated in cancers. Our findings demonstrate the importance of considering both environmental and intracellular regulation when interpreting tumour metabolism for diagnostic or prognostic purposes. To illustrate this, we demonstrate the potential impact of this multi-factorial regulation on the kinetics of radiolabelled glucose analogues, used in positron emission tomography (PET). (c) 2008 Elsevier Ltd. All rights reserved.
\end{abstract}

\section{Introduction}

Cancer is a devastating, multi-factorial disease which accounts for over 150,000 deaths per year in the United Kingdom alone. There is thus an ever-increasing effort to understand the nature of the disease and improve diagnosis and treatment of it. However, the complexity of cancer presents an ongoing problem; the sheer number of interactions renders the attribution of individual phenomena to a particular cause extremely difficult. In this paper, we present and investigate a model that describes the interplay between hypoxia-dependent and -independent regulation of glucose metabolism. Using numerical techniques, we explore the effects of one such interaction between the microenvironment and intracellular signalling on the glycolytic response of tumour tissue. Implications for imaging are discussed.

The metabolism of glucose in tumours is markedly different from that of most tissues, and much debate exists regarding the degree to which the origin of this can be attributed to either environmental or cellular factors. In normal cells, glycolysis, the production of two molecules of pyruvate from a molecule of glucose, serves as the initial step in aerobic metabolism. In the presence of oxygen, pyruvate is converted to water and carbon dioxide, producing the cell's energy currency, adenosine triose phosphate (ATP), in the process. In the absence of oxygen however, anaerobic metabolism takes over, and energy is solely produced by glycolysis. Glycolysis alone produces 18 times less

\footnotetext{
* Corresponding author. Tel.: +441865283063.

E-mail address: ckelly@robots.ox.ac.uk (C. Kelly).
}

ATP per glucose molecule than aerobic respiration, thus in normal tissue anaerobic metabolism is usually only a temporary measure, associated with increased proliferation and inflammation. In tumours, however, glycolysis is the norm, with the energetic inefficiency compensated by a several-fold increase in glucose flux. This phenomenon is routinely exploited for tumour imaging in the clinic through the radiolabelled analogue of glucose, 18-fluorodeoxyglucose (FDG) in conjunction with positron emission tomography (PET) (Czernin and Phelps, 2002; Gambhir, 2002). Quantitative analysis of FDG-PET data conventionally takes one of two forms: (i) calculation of a single normalised value (the standardised uptake value, or SUV) from FDG activity accumulated over a single time period, or (ii) estimation of kinetic parameters including the metabolic rate of glucose $\left(M R_{G l u}\right)$ and the volume of distribution from compartment modelling or graphical analysis of dynamic data. Both the SUV and kinetic parameters may be used to detect increased tumour glycolysis, but in themselves offer no further causal explanation.

In addition to the increased energy requirements of proliferating cells, the tumour microenvironment can also play a role in the regulation of glycolysis. In early stages of development, most tumours lack a vascular supply, and the supply of oxygen is limited by its diffusion distance. As the tumour continues to grow, cells proliferate into regions with little oxygen, becoming hypoxic. Hypoxia in tumours is associated with a lack of response to radiotherapy and is predictive of malignant progression. At the level of the individual cell, hypoxic stress is potentially very harmful, so a protective response is necessary. This is mediated by hypoxia-inducible factor-1 (HIF-1), a transcription factor which acts as a cellular oxygen sensor. Under conditions of 
hypoxia, HIF-1 rapidly translocates to the nucleus, where it binds to the promoters of genes with consensus binding site sequences, known as hypoxia-response elements (HREs), facilitating their transcription. The genes which have been found to contain HREs encode enzymes involved in many processes of stress response, including glycolysis (Semenza et al., 1994). Indeed, strong correlations between oxygen tension and the uptake of the FDG have been observed in a number of cell lines, illustrating this (Clavo et al., 1995; Burgman et al., 2001). However, the relationship between glycolysis and hypoxia is a variable one; one group that reported good correlation between FDG activity, hypoxia and HIF- $1 \alpha$ expression in glioma noted a lack of correlation in nonsmall cell lung cancer (Cher et al., 2006; Cherk et al., 2006).

HIF-mediated regulation of glycolysis extends well beyond hypoxia. In addition to being an oxygen sensor, HIF-1 also acts as a downstream effector of certain signalling cascades, such as that of endothelial growth factor (EGF). Growth factors such as EGF and platelet-derived growth factor (PDGF) also stimulate glycolysis, via the Ras/PI3-K/PTEN/Akt pathway. Although in normal tissue, stimulation via growth factors occurs infrequently, in tumours pathway activation is frequently dysregulated. Recent studies suggest that HIF-1 mediates the signal from the Akt; Akt is responsible for the activation of the HIF- $1 \alpha$ translation enhancers, mTOR and p70S6K1 (Saitoh et al., 2002; Peterson et al., 2000). Fig. 1 is a deliberately simplified account of the hypoxiadependent and -independent HIF-mediated regulation of glycolysis. Signals received by receptor tyrosine kinases (RTKs) such as those receptors for EGF and PDGF stimulate activation of the Ras/PI3-K/PTEN/Akt pathway (hereafter referred to as Akt). mTOR, through activation of the kinase p70S6K1, leads to increased translation of the HIF- $1 \alpha$ subunit. At the same time, cellular oxygen regulates the degradation of HIF- $1 \alpha$, through the action of HIF prolyl hydroxylase (HPH). Intact HIF- $1 \alpha$ dimerises with HIF-1 $\beta$ /ARNT to form the active transcription factor. This enhances transcription of HRE-containing genes, including those involved in glycolysis.

Whilst it is generally accepted that glycolytic regulation is multi-factorial, there is relatively little understanding of how the different mechanisms that the individual factors employ might interact to control glycolysis. Mathematical modelling has the potential to provide a precise account of this process; indeed, a number of models have been developed which describe the tumour microenvironment, at differing levels of complexity (Thomlinson and Gray, 1955; Smallbone et al., 2005, 2007). Concentration profiles of oxygen in 1-D tissue of uniform metabolism, supplied by a single vessel, were first calculated analytically by Thomlinson and Gray (1955) using partial differential equations (PDEs). This work enabled calculation of the maximum diffusion distance of oxygen, correctly predicting the maximum radius of tumour cords. Since then, models of spheroids have been developed that correctly predict the growth of tumours in response to spatial profiles of essential nutrients. The model of Venkatasubramanian et al. (2006) describes glucose metabolism in relation to cellular oxygen concentration, assuming that the maximum glycolytic capacity is a fixed value; the rate of glycolysis is affected only by glucose and oxygen concentrations. This representation neglects both the hypoxic response of the cell and the oncogenic activity. Smallbone et al. (2005) come closer to addressing this by employing two rates of glycolysis; representing normal and 'abnormal' (i.e. increased) metabolism, although this does not consider specific oncogenes. With regard to the upregulation of glycolysis in hypoxia, as far as we are aware there exists no previous literature.

In this paper, we build upon previous models of molecular concentrations in the tumour microenvironment (e.g. Smallbone, Baxter and Jain), and incorporate the effects of intracellular signalling, through the specific inclusion of HIF-1 as a mediator of hypoxia and growth-factor signalling. Given the general absence of experimentally determined parameter values, the model is necessarily simplified, but nonetheless captures the overall dynamics of the system. We find that the model replicates the close relationship between oxygen and glycolysis. Moreover, we find that, within our modelling framework, we might assess the potential role of the PET tracer FDG as a non-invasive marker of hypoxia.

\section{Model development}

In our model, we assume an avascular tumour of constant size, forcing nutrient supply and waste removal to occur via the tumour boundary. The spatiotemporal distributions of molecules are governed by individual diffusional and biochemical properties. Following previous authors, we assume spherical symmetry, allowing us to perform analysis of the general tissue dynamics in response to differing microenvironmental conditions. We assume that bulk molecular transport is via diffusion. Neglecting the influence of convection on tracer delivery can be justified by the many observations of low convection within tumours, due to the absence of functioning lymphatics (Padera et al., 2002; Zeidman et al., 1955) and the higher permeability of tumour blood vessels (Baxter and Jain, 1991). We further assume that reactions follow Michaelis-Menten-type kinetics; i.e. rates are concentration-dependent whilst also saturable.

The framework for our model of HIF-mediated glycolysis is depicted in Fig. 2. Here we position HIF at the centre of our system, receiving signals from Akt and oxygen and transmitting signals to enhance glycolysis and FDG binding. Note that we have deliberately reduced the model from that depicted in Fig. 1, omitting many of the intermediate steps; this serves to minimise the number of microenvironmental and expression parameters required to solve the model and to clarify relationships between pathways. Evidently, this modelling strategy runs the risk of omitting missing key information from the model; however, we contend that we have maintained all major relationships.

Let $O$ denote the partial pressure of oxygen, $H$ the intracellular concentration of HIF-1, and $G$ and $F$ the tissue concentrations of glucose and FDG, respectively. We assume that oxygen diffuses freely through intra- and extra-cellular space equally at a rate $D_{0}$ and that glucose and FDG diffuse freely from the vasculature and within the extracellular space at an identical rate, $D_{G}$. HIF-1, as a

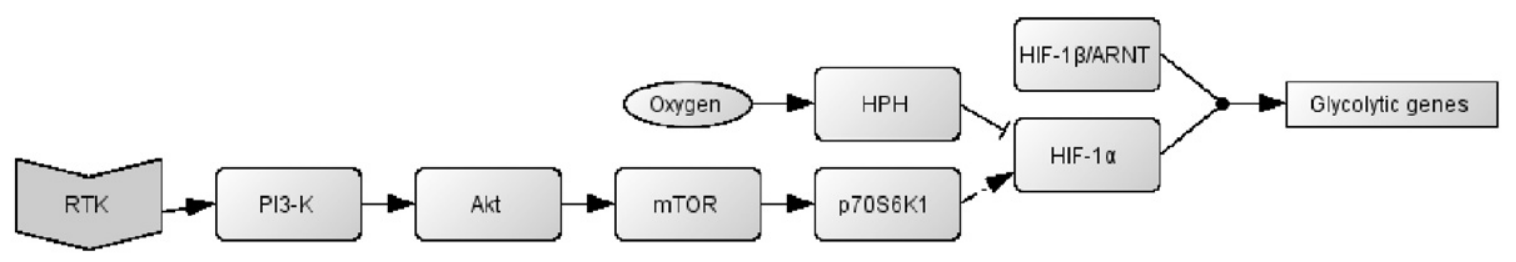

Fig. 1. Simplified network model of HIF-mediated glycolysis. The seemingly disparate physiological and biological influences both exert their effect through HIF-1. 


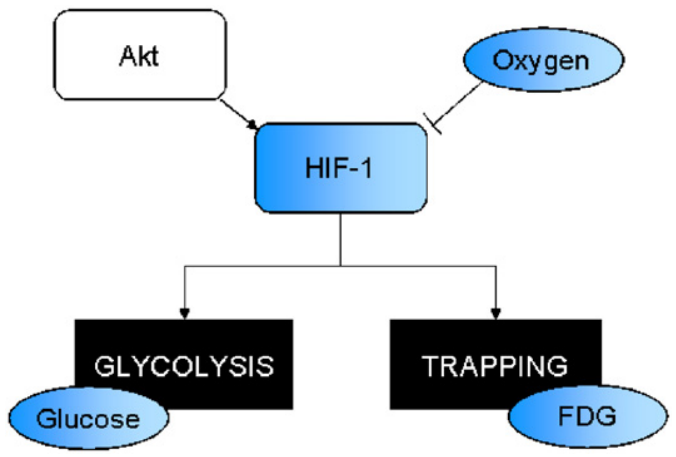

Fig. 2. Diagrammatic representation of HIF-centric model. Model variables are depicted in blue. Processes are shown in black squares. Small molecules and proteins are depicted by ovals and round-cornered rectangles, respectively. FDG, whilst an analogue of glucose, does not undergo glycolysis; instead it becomes trapped within the cell.

transcription factor, does not diffuse through tissue; rather, it is produced and metabolised intracellularly. The depletion rates of free glucose (i.e. cellular uptake and consumption) and FDG (i.e. cellular uptake and trapping), denoted by $V_{G}$ and $V_{F}$, respectively, are assumed dependent on HIF-1 concentration. Under these assumptions, we may translate Fig. 2 into the following series of coupled equations:

$\frac{\partial O(x, t)}{\partial t}=D_{O} \nabla^{2} O-d_{O}\left(\frac{O}{K m_{O}+O}\right)$

$\frac{\mathrm{d} H}{\mathrm{~d} t}=r_{H}^{\star}\left(\frac{H_{\max }-H}{H_{\max }}\right)-d_{H}\left(\frac{O}{K m_{H}+O}\right) H$

$\frac{\partial G(x, t)}{\partial t}=D_{G} \nabla^{2} G-v_{G}$

$\frac{\partial F(x, t)}{\partial t}=D_{G} \nabla^{2} G-v_{F}$

where $r_{H}^{\star}$ and $d_{H}$ denote the maximum rates of HIF-1 production and degradation, respectively, $d_{O}$ is the maximum rate of oxygen consumption, and $\mathrm{Km}_{O}$ the concentration at which consumption is half-maximal. Here, we assume that the hypoxia-responsive effects of HIF-1 on oxygen concentration, such as angiogenesis, are not seen within the time frame of a PET scan. Production of HIF-1 is dependent on both the intrinsic capacity to synthesise, given by $r_{H}^{\star}$, and a maximal cellular concentration, $H_{\max }$ (Wang and Semenza, 1993), which may not be succeeded. Degradation of HIF- $1 \alpha$ is dependent on the local oxygen concentration with $\mathrm{Km}_{\mathrm{H}}$ denoting the oxygen tension at which the rate of degradation is half-maximal.

The influence of Akt activity on HIF- $1 \alpha$ is effected through $r_{H}^{\star}$. It is likely that regulation results in a range of potential levels of Akt; however, since there is as yet no quantitative data on Akt protein levels in relation to its regulators, we make the simplifying assumption that Akt exists in two states, being either active or inactive. In the active state, $r_{H}^{\star}=r_{H}^{A}$ (Active) whilst in the inactive state $r_{H}^{\star}=r_{H}^{I}$ (Inactive). Production in the active state may be thought of as a multiple of the normal production rate-that is Akt acts as a gain term-where the increase in $r_{H}^{\star}$ is given by

$r_{H}^{A}=\alpha r_{H}^{I}$

We now define the rates of glucose consumption and FDG trapping, $v_{G}$ and $v_{F}$, respectively. FDG and glucose are sufficiently analogous that whilst they have very similar kinetics, they are also in competition for the transporters and enzymes. Let the shared maximum reaction velocity for both molecules be $d_{G}$ and the concentration at which this velocity is half-maximal be $\mathrm{Km}_{G}$ and
$K m_{F}$ for glucose and FDG, respectively. We know that under near anoxia (when we presume HIF-1 levels to be maximal) glycolysis increases 2.5-fold as a result of increased enzyme levels (Burgman et al., 2001). As no further descriptive qualitative or quantitative data exist, we postulate that the reaction rate increases linearly with $H$ and bound by a factor of $k_{H}+1$ when $H$ is maximal. Assuming that FDG levels are negligible in comparison to glucose (as is the rule for PET studies), the rates of glucose consumption and FDG trapping may be defined:

$v_{G}=d_{G} \frac{G}{\left(K m_{G}+G\right)}\left(1+k_{H} H\right)$

$v_{F}=d_{G} \frac{F}{\theta\left(K m_{G}+G\right)}\left(1+k_{H} H\right)$

where

$\theta=\frac{K m_{F}}{K m_{G}}$

Eq. (7) suggests that retention of FDG is linear in $F$, and inversely related to the concentration of tissue glucose. This relationship is taken into account in the clinic, where patients are required to fast prior to imaging with FDG, in order to reduce blood glucose levels and minimise competition, resulting in increased FDG contrast.

We note that some of the quantities in our model are present at concentrations of different orders of magnitude; e.g. glucose and FDG. Furthermore, as a result of inherent difficulty in quantification, some of the parameters for HIF-1 are unknown (e.g. $H_{\max }$ ) or hard to estimate $\left(r_{H}^{\star}\right)$. We thus non-dimensionalise the molecular concentrations with respect to their maximum concentrations and define the dimensionless numbers:

$h=H / H_{\max }, \quad o=O / O_{\max }$

$g=G / G_{\max }, \quad f=F / F_{\max }$

where $H_{\max }$ is unknown, $O_{\max }$ and $G_{\max }$ are the blood plasma concentrations of oxygen and glucose, respectively (assumed constant over the period of study), and $F_{\max }$ is the maximal plasma concentration of FDG.

\section{Numerical results}

\subsection{Methods}

All simulations were performed in Matlab (Matlab, 2005), using finite-difference methods (spatial grid size $\Delta=1 \mu \mathrm{m}$ ). Parameter values used are set out in Table 1. As boundary conditions, in the plasma we assume glucose and oxygen to be fixed at $5 \times 10^{-4} \mathrm{M}$ and $40 \mathrm{mmHg}$, respectively. The form of the plasma input function for FDG (specifically $f$ ) is an exponential decay function with a dimensionless maximum concentration of 1 and a half-life of $15 \mathrm{~min}$. At the distal edge of the tissue we assume zero flux. As HIF levels take several hours to reach maximal levels, we first bring the system $(O, G$ and $H)$ to equilibrium prior to introducing FDG at time $\phi=6 \mathrm{~h}$.

\subsection{Spatial profiles}

We first consider the case where the influence of Akt on HIF-1 is null, i.e. $\alpha=0$. Fig. 3 shows spatial profiles of oxygen, HIF-1, glucose and FDG, 120 min after FDG is added. In agreement with the work of Thomlinson and Gray (1955), oxygen concentration decreases rapidly with increasing distance from the source $(0 \mu \mathrm{m})$. This is accompanied by a corresponding increase in HIF-1, which is rapid initially, but plateaus within approximately $150 \mu \mathrm{m}$. Like oxygen, glucose also falls with distance from the source; however, 
Table 1

Parameter values used in the model

\begin{tabular}{|c|c|c|c|}
\hline Parameter & Value & Units & Reference \\
\hline$r_{H}$ & $1.21 \times 10^{-4}$ & $\mathrm{~s}^{-1}$ & Estimated; consistent with Wang and Semenza (1993) \\
\hline$d_{H}$ & $2.3 \times 10^{-3}$ & $\mathrm{~s}^{-1}$ & Estimated from Jewell et al. (2001) \\
\hline$K m_{H}$ & 2 & $\mathrm{mmHg}$ & Dasu et al. (2003) \\
\hline$d_{0}$ & 15 & $\mathrm{mmHg} \mathrm{s}^{-1}$ & Dasu et al. (2003) \\
\hline$K m_{O}$ & 2 & $\mathrm{mmHg}$ & Dasu et al. (2003) \\
\hline$D_{0}$ & $2 \times 10^{-9}$ & $\mathrm{~m}^{2} \mathrm{~s}^{-1}$ & Dasu et al. (2003) \\
\hline$d_{G}$ & $5 \times 10^{-8}$ & $\mathrm{Ms}^{-1}$ & Brock et al. (2000) \\
\hline$K m_{G}$ & $3.9 \times 10^{-5}$ & M & Muzi et al. (2001) \\
\hline$\theta$ & 1.86 & & Estimated from Muzi et al. (2001) \\
\hline$k_{h}$ & 1.5 & & Estimated from Burgman et al. (2001) \\
\hline$D_{G}$ & $5.5 \times 10^{-11}$ & $\mathrm{~m}^{2} \mathrm{~s}^{-1}$ & Casciari et al. (1988) \\
\hline
\end{tabular}

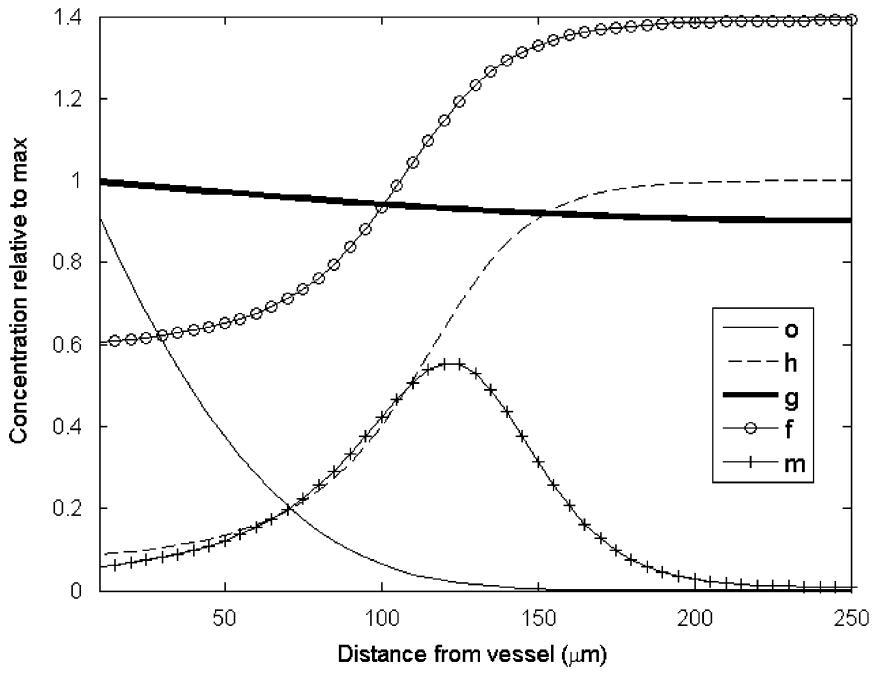

Fig. 3. Spatial profile of molecular quantities (relative to max values: cellular for HIF-1, plasma for all others), in the absence of oncogenic activity, 120 min postadministration of FDG.

as observed by Smallbone et al. (2005), consumption is less intense, thus levels remain above zero across the domain. As expected, FDG levels follow those of HIF-1.

We first consider the influence of positive Akt influence on glucose. Fig. 4 shows the spatial profile of glucose, at steady state, in relation to increasing influence of Akt activity (as defined by increasing $\alpha$ ). Although a change in profile is observed, this does not at first suggest that Akt activity should have a significant effect on FDG.

\subsection{Imaging outcome}

We now consider the influence of HIF-mediation on FDG imaging outcome. Fig. 5 shows temporal profiles, or tissue activity curves (TACs), of FDG concentration in the absence of Akt activity, at different distances from the vessel, corresponding to different steady-state oxygen tensions; $24 \mathrm{mmHg}$ at $25 \mu \mathrm{m} ; 1 \mathrm{mmHg}$ at $125 \mu \mathrm{m} ; 0 \mathrm{mmHg}$ at $200 \mu \mathrm{m}$. As FDG is retained in greater quantities in the hypoxic tissue with respect to the normoxic tissue, contrast is observed, reaching a maximum after approximately $1 \mathrm{~h}$. This corresponds to the imaging time used clinically for best contrast.

We now turn to examine the influence of pathway activity, via $\alpha$, on FDG retention. In Fig. 6, the spatial profiles of FDG at $120 \mathrm{~min}$ are shown for a range of values for $\alpha$; we see that as $\alpha$ increases,

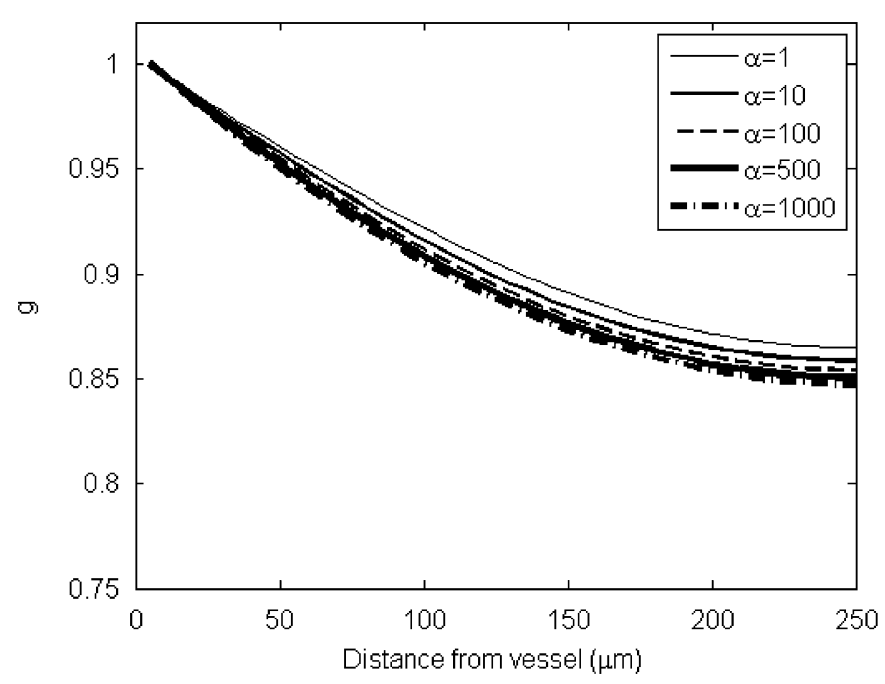

Fig. 4. Effect of $\alpha$ on spatial distribution of $g$ at steady state.

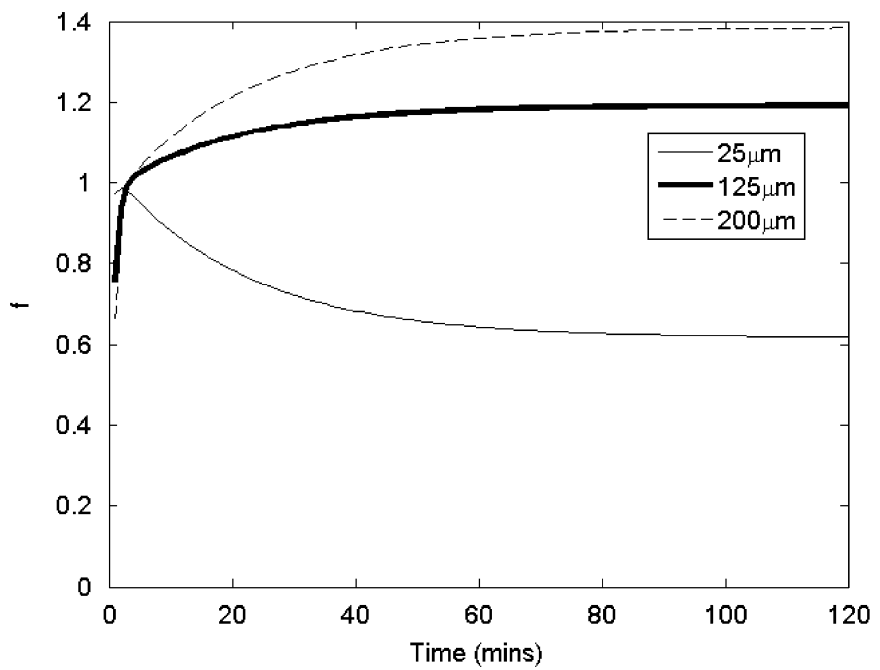

Fig. 5. Temporal evolution of $f$, at 25,125 , and $200-\mu \mathrm{m}$ from the vessel.

the difference between $f$ at 10 and $100 \mu \mathrm{m}$ decreases. The outcome of this from an imaging standpoint would be a reduced hypoxia to normoxia contrast.

Measuring image contrast at a single time point is informative, but underutilises the full biological information available in 


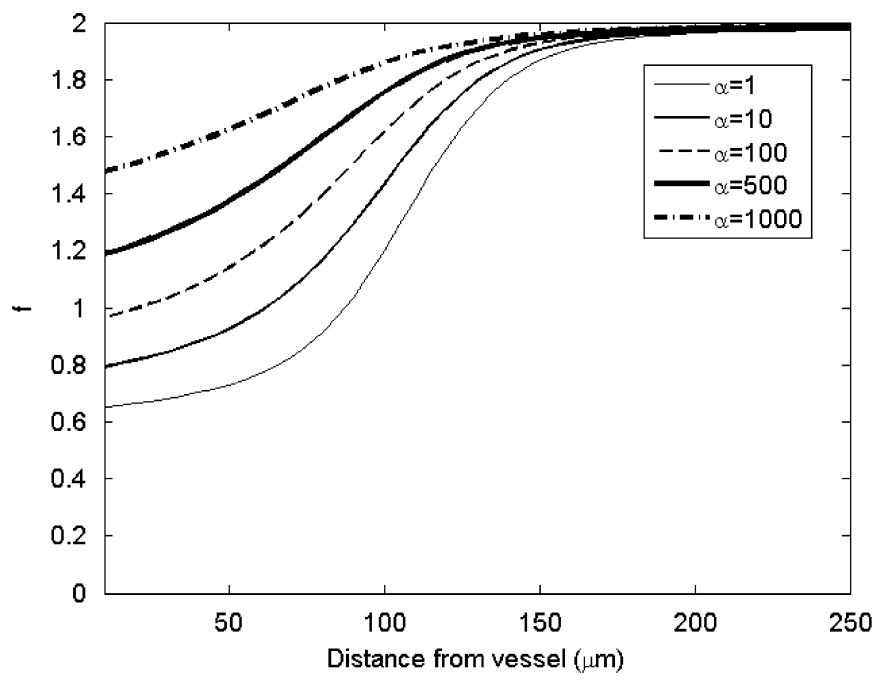

Fig. 6. Effect of $\alpha$ on spatial distribution of $f$ at $120 \mathrm{~min}$.

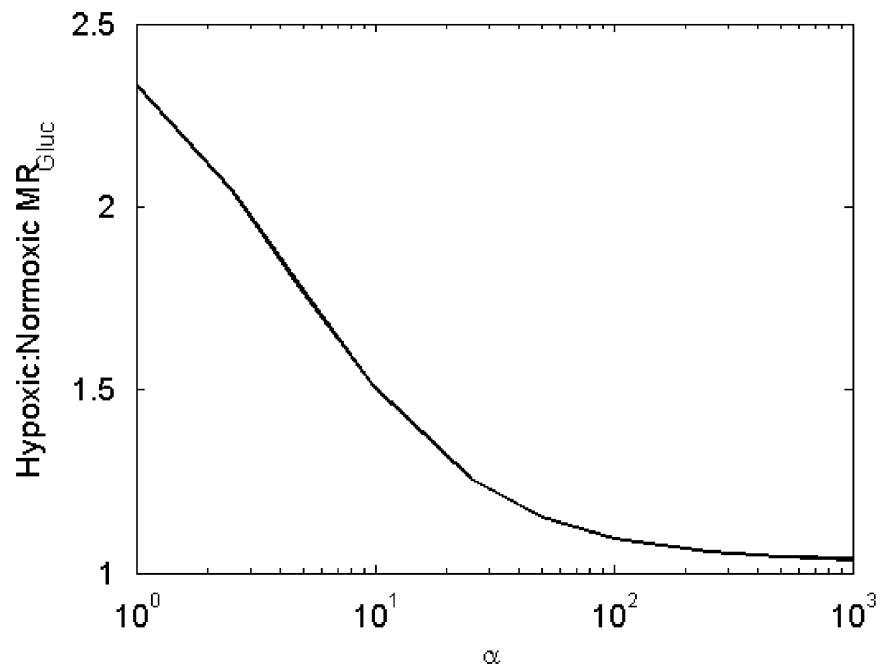

Fig. 7. Effect of $\alpha$, on the ratio of hypoxic to normoxic $M R_{G l u}$ s. Note log scale.

temporal (TAC) data. We now calculate the metabolic rate of glucose $\left(M R_{G l u}\right)$ from normoxic and hypoxic TACs using a twotissue compartment model; details of this calculation are given elsewhere (Phelps et al., 1979). We then define the ability of FDG to distinguish between hypoxia and normoxia as the ratio between metabolic rates of glucose in hypoxic and normoxic tissues, i.e.

$M R_{G l u}^{H / N}=M R_{G l u}^{\text {Hypoxic }} / M R_{G l u}^{\text {Normoxic }}$

where normoxic and hypoxic TACs are taken at 25 and $100 \mu \mathrm{m}$ from the vasculature, respectively. In Fig. 7, we see how the hypoxic parameter $M R_{G l u}^{H / N}$ varies with changes to the influence of Akt. As $\alpha$ increases, $M R_{G l u}^{H / N}$ falls; the loss of hypoxic:normoxic contrast is small at low values of $\alpha$, but becomes almost total as $\alpha$ surpasses 1000 . The burning question now concerns the feasibility of the level of increased production. The mechanism of action of Akt, via mTOR, suggests that this may indeed be possible. mTOR acts to enhance translation in an mRNA-specific manner, to a 'disproportionate and dramatic' extent (Gingras et al., 2001). Whilst no data exist to quantify the increase in HIF- $1 \alpha$ as a result of mTOR, it may well reach the several orders of magnitude that are necessary.

\section{Discussion and future work}

In this work, we have extended previous models of tumour metabolism in response to the microenvironment, and developed a framework to investigate the relative influence of hypoxiadependent and -independent regulators of glycolysis. In particular, we model (i) the explicit relationship between oxygen and glycolysis, via HIF-1, and (ii) the effects of constitutive upregulation of the PTEN/PI3-K/Akt pathway on cellular levels of HIF-1. What is particularly novel about our approach is the application to the understanding of PET image formation.

Previous models of glucose metabolism have only considered oxygen in its role as the final electron acceptor in mitochondrial aerobic metabolism; we explicitly take into account the cellular response to hypoxic stress and the associated increased expression of glycolytic enzymes associated. In consideration of the two highly different methods of HIF-1 regulation, we find that the influence of Akt on production should be several orders of magnitude to have a significant effect on HIF-1 levels in normoxic conditions. This might suggest that the effect of Akt is minimal; however, the amplifying nature of intracellular signalling would enable such an effect. Furthermore, Akt acts by increasing translation of HIF- $1 \alpha$ protein; translational enhancement can be significant, thus the effect required is wholly feasible.

In the absence of Akt activity, we find that our model replicates previous observations in the literature of a close relationship between cellular oxygen and glycolysis. This suggests a potential role for the imaging analogue of glucose, FDG, as a non-invasive marker of hypoxia. Indeed, using our model to simulate the uptake and retention of FDG in normoxic and hypoxic tissues, we find that contrast between the two tissue types is observed.

We find that the influence of Akt on the steady-state concentration of glucose is minimal and thus might expect there to be a similar lack of influence on FDG kinetics; however, this is not the case. One potential explanation is that the rate of consumption of glucose relative to the blood levels of glucose is relatively small and thus glucose levels remain high; in the case of FDG, however, which is administered in trace amounts, the same consumption rate equates to a relatively higher proportion of the molecular pool that is metabolised.

Our hypothesis that dysregulation of Akt may adversely affect the imaging outcome for FDG is borne out by the results of our simulations. Using the ratio of $M R_{G l u}$ in hypoxic tissue to that in normoxic tissue as a measure of the ability of FDG to distinguish between the two tissue types, we show that as the influence of Akt on HIF- $1 \alpha$ production increases, there is an associated loss of contrast, as demonstrated in Fig. 7. As we commented earlier, the quantitative effect of Akt on production must be two to three orders of magnitude greater to stabilise HIF-1 levels. Whilst the feasibility of this may be called into question if upregulation were via global enhancement of protein synthesis, the mRNA-specific mechanism of the Akt-regulated effector mTOR is such that translational enhancement could well be of this order. If a tumour is FDG-avid and simultaneously Akt-active, there would be a reduced certainty in the cause of this being tumour hypoxia. Furthermore, cancers consist of clonal populations, often with heterogeneous genotypes, further confounding any hypoxic signal. It would follow then that if dysregulation of Akt activity were a likelihood, FDG would be wholly unsuitable as a marker of hypoxia. The PTEN/PI3-K/Akt pathway has been shown to be dysregulated in a high number of tumour types (Faivre et al., 2006), including breast, colon and head and neck carcinomas, 
suggesting that use of FDG as a surrogate marker of hypoxia should be with extreme caution. In general, these findings demonstrate the importance of considering the influence of tumour genetics on a process whose regulation is associated with microenvironmental influences.

It may be objected that pathways involved in the regulation of glucose metabolism have been grossly simplified, even neglected. However, to model the entire network of molecules involved in some way with glycolysis would require that the values of thousands of parameters be determined. Additionally, although it may be the case that a large number of pathway components contribute to the behaviour of the system, there is much redundancy, and it is difficult to extract individual influences. Rather, we feel that it makes more sense to limit the number of components to the bare minimum that is required to capture the behaviour of the system observed experimentally. Furthermore, the framework that we have developed may easily be extended to incorporate more complex examples than that presented here. We are also aware that some of the assumptions made in the formulation of model equations require validation; for example, the quantitative effect of constitutive Akt (or other pathway constituents) activity on HIF-1 production should be made clear. Furthermore, to fully validate our approach, it will be necessary to identify whether the predictions made by the model are borne out in reality. We are presently validating our assumptions and predictions in vitro, imaging HIF-1 expression and FDG uptake in tumour spheroids where Akt is constitutively active, in the presence and absence of the Akt inhibitor, LY294002.

In this paper, we have demonstrated the general utility of our approach; a framework suitable for investigating the impact of multi-factorial regulation on imaging outcome. However, the model in its current form is limited in its application as it describes HIF- $1 \alpha$, oxygen, glucose and FDG concentration as a function of distance from a single blood vessel only. To fulfil our goal of modelling in vivo tissue, we intend to extend our model to incorporate a more realistic vascular architecture similar to that used to describe the effects of vessel density on Fmiso kinetics (Kelly and Brady, 2006). We are also working towards extending our model in order to investigate alternative tracers for hypoxia, as well as other characteristic cancer indicators, such as angiogenesis. Our ultimate goal is the intelligent assessment and design of PET tracers and imaging protocols for specific indications.

\section{References}

Baxter, L.T., Jain, R.K., 1991. Transport of fluid and macromolecules in tumors. III. Role of binding and metabolism. Microvasc. Res. 41, 5.

Brock, C.S., Young, H., O'Reilly, S.M., et al., 2000. Early evaluation of tumour metabolic response using [18F]fluorodeoxyglucose and positron emission tomography: a pilot study following the phase II chemotherapy schedule for temozolomide in recurrent high-grade gliomas. Br. J. Cancer 82, 608.
Burgman, P., ODonoghue, J.A., Humm, J.L., 2001. Hypoxia-induced increase in FDG uptake in MCF7 cells. J. Nucl. Med. 42, 170.

Casciari, J.J., Sotirchos, S.V., Sutherland, R.M., 1988. A modeling approach for quantifying tumor hypoxia with [F-18]fluoromisonidazole PET time-activity data. Cancer Res. 48, 3905.

Cher, L.M., Murone, C., Lawrentschuk, N., Ramdave, S., Papenfuss, A., Hannah, A. OḰeefe, G.J., Sachinidis, J.I., Berlangieri, S.U., Fabinyi, G., Scott, A.M., 2006. Correlation of hypoxic cell fraction and angiogenesis with glucose metabolic rate in gliomas using $18 \mathrm{~F}$-fluoromisonidazole, $18 \mathrm{~F}-\mathrm{FDG}$ PET, and immunohistochemical studies. J. Nucl. Med. 47, 410.

Cherk, M.H., Foo, S.S., Poon, A.M.T., Knight, S.R., Murone, C., Papenfuss, A.T., Sachinidis, J.I., Saunder, T.H.C., O'Keefe, G.J., Scott, A.M., 2006. Lack of correlation of hypoxic cell fraction and angiogenesis with glucose metabolic rate in non-small cell lung cancer assessed by $18 \mathrm{~F}$-Fluoromisonidazole and 18F-FDG PET. J. Nucl. Med. 47, 1921.

Clavo, A.C., Brown, R.S., Wahl, R.L., 1995. Fluorodeoxyglucose uptake in human cancer cell lines is increased by hypoxia. J. Nucl. Med. 36, 1625.

Czernin, J., Phelps, M.E., 2002. Positron emission tomography scanning: current and future applications. Annu. Rev. Med. 53, 89.

Dasu, A., Toma-Dasu, I., Karlsson, M., 2003. Theoretical simulation of tumour oxygenation and results from acute and chronic hypoxia. Phys. Med. Biol. 48, 2829.

Faivre, S., Kroemer, G., Raymond, E., 2006. Molecular events resulting in the activation of the PI3K/AKT/mTOR pathway in human tumours. Nat. Rev. Drug Discov. 5, 671 .

Gambhir, S.S., 2002. Molecular imaging of cancer with positron emission tomography. Nat. Rev. Cancer 2, 683.

Gingras, A.C., Raught, B., Sonenberg, N., 2001. Control of translation by the target of rapamycin proteins. Prog. Mol. Subcell. Biol. 27, 143.

Jewell, U.R., Kvietikova, I., Scheid, A., Bauer, C., Wenger, R.H., Gassmann, M., 2001. Induction of HIF-1alpha in response to hypoxia is instantaneous. FASEB J. 15, 1312.

Kelly, C.J., Brady, J.M., 2006. A model to simulate tumour oxygenation and dynamic [18F]-Fmiso PET data. Phys. Med. Biol. 51, 5859.

Muzi, M., Freeman, S.D., Burrows, R.C., et al., 2001. Kinetic characterization of hexokinase isoenzymes from glioma cells: implications for FDG imaging of human brain tumors. Nucl. Med. Biol. 28, 107.

Padera, T.P., Kadambi, A., di Tomaso, E., et al., 2002. Lymphatic metastasis in the absence of functional intratumor lymphatics. Science 296, 1883.

Peterson, R.T., Beal, P.A., Comb, M.J., Schreiber, S.L., 2000. FKBP12-rapamycinassociated protein (FRAP) autophosphorylates at serine 2481 under translationally repressive conditions. J. Biol. Chem. 10, 7416.

Phelps, M.E., Huang, S.C., Hoffman, E.J., Selin, C., Sokoloff, L., Kuhl, D.E., 1979. Tomographic measurement of local cerebral glucose metabolic rate in humans with (18F) 2-fluoro-2-deoxy-D-glucose: validation of method. Ann. Neurol. 6, 371.

Saitoh, M., Pullen, N., Brennan, P., Cantrell, D., Dennis, P.B., Thomas, G., 2002. Regulation of an activated S6 kinase 1 variant reveals a novel mammalian target of rapamycin phosphorylation site. J. Biol. Chem. 277, 20104.

Semenza, G.L., Roth, P.H., Fang, H.M., Wang, G.L., 1994. Transcriptional regulation of genes encoding glycolytic enzymes by hypoxia-inducible factor 1 . J. Biol. Chem. 269, 23757.

Smallbone, K., Gavaghan, D.J., Gatenby, R.A., Maini, P.K., 2005. The role of acidity in solid tumour growth and invasion. J. Theor. Biol. 21, 476.

Smallbone, K., Gavaghan, D.J., Maini, P.K., Brady, J.M., 2007. Quiescence as a mechanism for cyclical hypoxia and acidosis. J. Math. Biol. 55, 767.

Thomlinson, R.H., Gray, L.H., 1955. The histological structure of some human lung cancers and the possible implications for radiotherapy. Br. J. Cancer 9, 539.

Venkatasubramanian, R., Henson, M.A., Forbes, N.S., 2006. Incorporating energy metabolism into a growth model of multicellular tumor spheroids. J. Theor. Biol. 242, 440

Wang, G.L., Semenza, G.L., 1993. Characterization of hypoxia-inducible factor 1 and regulation of DNA binding activity by hypoxia. J. Biol. Chem. 268, 21513.

Zeidman, I., Copeland, B.E., Warren, S., 1955. Experimental studies on the spread of cancer in the lymphatic system. II. Absence of a lymphatic supply in carcinoma. Cancer 8, 123. 